\title{
A welcome of the Immunologic Research's new editors
}

\author{
Nicola Bizzaro ${ }^{1,2} \cdot$ Dieter Kabelitz $^{3}$ \\ Published online: 4 August 2021 \\ (c) The Author(s), under exclusive licence to Springer Science+Business Media, LLC, part of Springer Nature 2021
}

Traditionally, Immunologic Research focused on various aspects of basic and clinical immunology, including adaptive and innate immunity, molecular immunology and signaling, organ-specific immunity, and immunotherapies and diseases such as allergy and inflammation, immunodeficiencies and autoimmunity, transplantation and tolerance, and tumor immunology.

When taking on the role of Editors-in-Chief of Immunologic Research, we decided to still consider articles from the abovementioned immunologic topics. However, we will shift the focus more to studies that address molecular and mechanistic aspects and include new topics that focus on systems immunology, new technologies, and immunity to emerging infections. We will also invite studies that describe the effect of genetic alterations (i.e., in genetically altered animals, cell lines, or patients) on the immune system with only a minimal set of experiments that address the underlying mechanism. Moreover, to protect findings that were produced independently by an individual laboratory, we will fast-track submissions on a topic that another group has recently published.

However, it is also necessary to reflect on how the editorial activity of medical journals, particularly those in the field of immunology, is evolving and has changed over the years.

The need to bring the evidence quickly to the scientific community's attention has long since changed the traditional process in the publication of research results in several respects, also thanks to the technological support of the

Nicola Bizzaro

nic.bizzaro@gmail.com

Dieter Kabelitz

dietrich.kabelitz@uksh.de

1 Laboratory of Clinical Pathology, San Antonio Hospital, Tolmezzo, Italy

2 Azienda Sanitaria Universitaria Integrata, Udine, Italy

3 Institute of Immunology, Christian-Albrechts University of Kiel and University Hospital Schleswig-Holstein Campus Kiel, Kiel, Germany
Internet. The first aspect is the recent multiplication of open access publications (some of them free of most copyright and licensing restrictions) that have reached over 12,500 journals publishing $60 \%$ of biomedically related articles [1]. A second element regards the connected phenomenon of predatory journals, of which some blacklists are available $[2,3]$. Predatory publishing is defined as a publishing model that mainly provides open access and asks for a publication fee. However, in contrast to a legitimate scientific publisher, the predatory journals are not listed in PUBMED and most ashamedly do not provide a controlled peer review [4].

Another recently surfaced aspect is the accelerated peer-review process. Following the COVID-19 pandemic, research times have radically shortened and the traditional way for acquiring evidence and publishing results has profoundly changed. Starting from March of last year, under the pressure of the pandemic, the traditional times and methods have jumped: some important journals have adopted the mechanism of an internal review, i.e., the evaluation of articles only by the editors, for the rapid publication of letters or short communications; Nature requested the cooperation of researchers outside its Editorial Board for accelerated reviews; eLife asked young researchers to take part in the review processes; the Royal Society Open Science has revised its Registered Report procedure intending to reduce the entire acceptance process to one week [5].

Although authors positively judge the acceleration of the publication process because it entails several advantages, especially for young researchers, such as rapid sharing of data and results and a proven primacy of discoveries, publishing non-peer-reviewed studies on pre-print servers has led to a stratospheric increase in data rather than knowledge, and increased caution in evaluating and diffusing information is therefore necessary.

Peer review in assessing the quality and originality of an article and propose or refuse its publication has several critical points, which can be summarized in: the appropriate choice of reviewers (real experience; subjectivity; sometimes incorrect conduct); some characteristics of the procedure (secrecy; ineffectiveness in detecting errors and plagiarism); 
and the slowness of the process (at least 3-4 months for publication; less than $7 \%$ of works accepted without major or minor revisions) [6].

In theory, speed and quality are not necessarily antithetical. However, in the practice of peer-review, the experience of the reviewers and the iterative process between reviewers, editors, and authors together guarantee an adequate filter to incorrect results and invalid interpretations. Nevertheless, it takes time. The simplification of the process, or a "leap" through submission of the manuscript to a pre-print server, which is required by some journals (e.g., Nature Communication), can help in terms of transparency and access to data in a rapid way, however the need for formal and thorough peer review remains vital [7]. As the not yet peer-reviewed pre-prints are also immediately accessible to the non-scientist community and the press, preliminary studies could lead to misleading information in broadcast, TV discussion panels, and newspapers. This is particularly serious in times of pandemics, when medical knowledge is instantly transferred to the clinical context and its therapeutic protocols, to the political context and subsequent health and economic measures, and even to the social context causing debates in newspapers, television, and social media [4].

Another issue closely related to the previous one is finding scientists willing to act as reviewers. Due to the enormous increase in medical journals, it is common to receive requests for reviewing papers almost daily. Therefore, most of these requests are necessarily declined, not only when specific expertise is lacking but also and above all, for lack of time. The pandemic has stolen energy and time from everyone, making it even more difficult to make oneself available for peer review. Notwithstanding these challenges, the editors of Immunologic Research intend to maintain a firm line on the principles and cornerstones that regulate publication and at the same time try to accelerate the process of peer-review and dissemination of the information produced by the research, without failing in their role as guarantors of robustness and sincerity of published data.

The entire newly formed Editorial Board and we are committed to bring our Journal to the next level by providing a platform for discussion and presenting controversial sights and establish it as a respected member in the family of all immunology journals. We hope that our rigorous editorial pre-screen and a thorough peer-review system and good bilateral communication with our authors will help us reach our goals.

\section{Compliance with Ethical Standards}

Conflict of interest The authors have no conflict of interest to declare.

\section{References}

1. Suber P. Open Access Overview. 2017 [Internet]. Available at: http://legacy.earlham.edu/ peters/fos/overview.htm

2. Silver A. Pay-to-view blacklist of predatory journals set to launch. Nature. 2017. https://doi.org/10.1038/nature.2017.22090.

3. Cukier S, Helal L, Rice DB, Pupkaite J, Ahmadzai N, Wilson M, et al. Checklists to detect potential predatory biomedical journals: a systematic review. BMC Med. 2020;18:104.

4. Marcus A, Oransky I. The science of the pandemic is moving at dangerous speed. Wired $2020 \mathrm{https}: / / \mathrm{www}$.wired.com/story/thescience-of-this-pandemic-is-moving-at-dangerous-speeds/

5. Nature Index. Rapid Registered Reports initiative aims to stop coronavirus researchers following false leads. [Internet]. Available at: https://www.natureindex.com/news-blog/rapid-registeredreport-coronavirus-aims-to-stop-researchers-following-false-resea rch-leads

6. Abdill RJ, Blekhman R. Preprint at bioRxiv. bioRxiv. 2019. https://doi.org/10.1101/515643.

7. Voss A. Statement on IJAA paper. International Society of Antimicrobial Chemotherapy 2020. [Internet]. Available at: https:// www.isac.world/news-and-publications/official-isac-statement

Publisher's Note Springer Nature remains neutral with regard to jurisdictional claims in published maps and institutional affiliations. 